OPEN ACCESS

Edited by:

Billy Sperlich,

University of Würzburg, Germany

Reviewed by:

Filipe Manuel Clemente,

Polytechnic Institute of Viana do

Castelo, Portugal

Fabio Augusto Barbieri, Universidade Estadual Paulista Júlio de Mesquita Filho (UNESP), Brazil

${ }^{*}$ Correspondence:

Luca P. Ardigò

luca.ardigo@univr.it

Specialty section:

This article was submitted to

Exercise Physiology,

a section of the journal

Frontiers in Physiology

Received: 09 May 2017 Accepted: 22 January 2018 Published: 06 February 2018

Citation:

Ardigò $L P$, Kuvacic G, lacono $A D$, Dascanio G and Padulo J (2018) Effect of Heart rate on Basketball

Three-Point Shot Accuracy.

Front. Physiol. 9:75.

doi: 10.3389/fphys.2018.00075

\section{Effect of Heart rate on Basketball Three-Point Shot Accuracy}

\author{
Luca P. Ardigò ${ }^{1 *}$, Goran Kuvacic ${ }^{2}$, Antonio D. lacono ${ }^{3}$, Giacomo Dascanio ${ }^{4}$ and \\ Johnny Padulo 2,5
}

'Department of Neurosciences, Biomedicine and Movement Sciences, School of Exercise and Sport Science, University of Verona, Verona, Italy, ${ }^{2}$ Faculty of Kinesiology, University of Split, Split, Croatia, ${ }^{3}$ Wingate Institute, Zinman College of Physical Education and Sport Sciences, Netanya, Israel, ${ }^{4}$ Associazione Dilettantistica Basket Club 7 Laghi Gazzada Schianno, Gazzada Schianno, Italy, ${ }^{5}$ Sport Science, University eCampus, Novedrate, Italy

The three-point shot (3S) is a fundamental basketball skill used frequently during a game, and is often a main determinant of the final result. The aim of the study was to investigate the effect of different metabolic conditions, in terms of heart rates, on 3S accuracy (3S\%) in 24 male (Under 17) basketball players (age $16.3 \pm 0.6$ yrs). 3S performance was specifically investigated at different heart rates. All sessions consisted of 10 consecutive 3Ss from five different significant field spots just beyond the FIBA three-point line, i.e., about $7 \mathrm{~m}$ from the basket (two counter-clockwise "laps") at different heart rates: rest (OHR), after warm-up (50\%HRMAX [50HR]), and heart rate corresponding to $80 \%$ of its maximum value (80\%HRMAX [80HR]). We found that $50 \mathrm{HR}$ does not significantly decrease $35 \%(-15 \%, P=0.255)$, while $80 \mathrm{HR}$ significantly does when compared to OHR $(-28 \%, P=0.007)$. Given that $50 \mathrm{HR}$ does not decrease $35 \%$ compared to $0 \mathrm{HR}$, we believe that no preliminary warm-up is needed before entering a game in order to specifically achieve a high 3S\%. Furthermore, 3S training should be performed in conditions of moderate-to-high fatigued state so that a high $3 \mathrm{~S} \%$ can be maintained during game-play.

\section{Keywords: fatigue, heart rate, task performance and analysis, sports, young}

\section{INTRODUCTION}

Due to its mixed physical-technical-tactical nature, basketball practice, like in other team sports, requires great attention to profiling the relevant physical and physiological characteristics of elite basketball players and, contextually, to determining the relationships between all of its features (e.g., metabolic demands) and the required technical skills. By investigating the typical game-driven physiological responses, recent research has widely determined the on-court activity patterns and the position-specific physical demands of this sport (Nikolaidis et al., 2014, 2015). In this regard, a body of studies has provided construct validity and reliability of physical assessment tests designed according to the running profile and activity patterns of the basketball players, by the inclusion of single or multiple changes of direction (Padulo et al., 2015b). Given the multi-faceted nature of basketball, whose performance success is a matter of physical, technical, and tactical ability, studies have been carried out as well regarding technical skills, such as the shooting task (Padulo et al., 2015a). A recent review (Okazaki et al., 2015) has reported that the ability to shoot an effective jump shot is critical for the player's success. Other findings are that players shoot more frequently in low-pressure and streaky situations (Csapo et al., 2015), and that compared to amateur players, professionals are able to shoot from greater distances and use more collective actions to find a shot 
position in which, possibly, the defensive pressure is lower (Ibáñez et al., 2009). Differences by playing position have been identified in short performers: point guards and power forwards shoot the most often and with the best accuracy in free-throw and two-point shots, whereas point guards shoot the most often and with the best accuracy in three-point shots (Ortega et al., 2006).

The above mentioned studies on factors influencing shooting performance have improved our understanding about the key factors targeted as fundamental for shooting performance accuracy. However, less information is available on the effect of fatigue, i.e., the variation of shooting accuracy over different exercise intensities, on the three-point shot (3S). In sport science, fatigue effects received attention, mainly because fatigue impacts overall athletes' performance (Faria et al., 2005). Harmful fatigue effects can diminish the function of a single whole-muscle, which leads to reduced muscle performance and therefore can decrease overall athletes' competition efficacy (Knicker et al., 2011). Fatigue can be described as a complex mechanism that involves both central and peripheral nervous system together with muscles (i.e., motor units; Ahmed, 2013). Poor perceptions, decisions, reactions, and resultant movement strategies are more likely to happen when athlete is in a fatigued state because central processing mechanisms and peripheral responses are compromised (Borotikar et al., 2008). In particular, the decisionmaking, considered as a high cognitive process, is closely related to fatigue and it can be said that a relation does exist between intensity and duration of the physical activity and this cognitive function (Abd-Elfattah et al., 2015). Commonly identified as an outcome of intense physical activity, fatigue has also been considered as a subjective experience, which can be described as a "sensation" (St Clair Gibson et al., 2003). Each individual has different sensations of fatigue and their generation is largely independent on the real biological state of the athlete, because brain uses the symptoms of fatigue as key regulators to protect body from potential damages (Noakes, 2012). Moreover, fatigue affects muscle strength, coordination, fine motor control, and movement patterns (Enoka and Stuart, 1992). Basketball players have high capability to move quickly, jump, and bounce the ball coordinating lower and upper limb movements (Cortis et al., 2011) and to achieve efficient basketball performance it is important to understand body adaptations and compensations under acute fatigue. Basketball players must be able to effectively perform specific tasks under conditions of physical fatigue that occurs during different training and game-play intensities (Kamandulis et al., 2013). Specifically about shooting performance, Barbieri et al. (2017) investigated fatigue effect on free-throw accuracy. They administered players a shuttle running fatiguing protocol and found postural control impairment but no free-throw accuracy decrease. Yet, by administrating such a protocol, they did not aim at achieving any reasonable heart ratewitnessed warm-up (Garrett and Kirkendall, 2000) and/or actual play (McInnes et al., 1995) metabolic intensity values.

Three-point shot performance is one of the main win determinants in elite basketball. Keeping a high $3 \mathrm{~S}$ percent accuracy (3S\%), especially during final minutes of close games, shows to be a key to success. Just as a reference, in the 2015-2016 season (regular season and playoffs), the NBA champions Cleveland Cavaliers shot-contested by players from the opposing team-3Ss in the first and last 2 min of regular quarters, and overtimes with a $3 \mathrm{~S} \%$ of 38.4 and $32.5 \%$, respectively ${ }^{1} 3 \mathrm{~S}$ is a common fundamental shot, which can be performed both when the players just come off the bench and when they are fatigued by previous actions. Knowledge about the effects of exercise intensity on 3S\% would be of great importance for both sport scientists and basketball practitioners (e.g., coaches and fitness trainers). Since no previous study has been performed on this topic (Padulo et al., 2015a), sport scientists could use any further information as reference data for future studies on a basketball task performance and analysis model. In addition, coaches and fitness trainers might benefit from such knowledge in order to develop suitable exercise interventions for optimizing shooting accuracy. Therefore, the aim of the present study was to examine the effect of different heart rates on successful $35 \%$. We hypothesized that increasing heart rate would pair with decreasing $3 \mathrm{~S} \%$.

\section{MATERIALS AND METHODS}

\section{Participants}

Twenty-four young (Under 17) basketball players (age 16.3 $\pm 0.6 \mathrm{yrs}$, height $180 \pm 6.1 \mathrm{~cm}$, mass $65.7 \pm 7.2 \mathrm{~kg}$, BMI $18.3 \pm 1.7 \mathrm{~kg} / \mathrm{m}^{-2}$, training experience $8.7 \pm 2.6 \mathrm{yrs}$ ) were recruited from Associazione Dilettantistica Basket Club 7 Laghi Gazzada Schianno teams. All players, in addition do their weekly practice, participated in the seasonal championship made up of a regional phase, an inter-regional phase, and national 16team finals. Inclusion criteria to participate in the study were: (i) participation in at least $85 \%$ of the previous season training sessions, (ii) regularly participating in the previous competitive season, (iii) having a valid sport medical certification, and (iv) being healthy (no pain or injury) and clear of any drug consumption. Participants refrained from drinking alcohol or caffeine-containing beverages for $24 \mathrm{~h}$, and did not eat for $3 \mathrm{~h}$, prior to testing to reduce any interference on the experiment. Each participant completed all trials in the same time period of the testing days (during the pre-season) and under the same climate conditions $\left[4-7\right.$ p.m., $23.2 \pm 0.6^{\circ} \mathrm{C}$ temperature and $55.3 \pm 1.8 \%$ relative humidity (i.e., day times and climate conditions similar to real game-play)], to eliminate any influence of circadian variation. All tests were performed on a regular indoor basketball court and the participants wore their official basketball uniforms. Participants gave their assent, and written consent was obtained from the participants' parents/guardians after being thoroughly informed about the purpose, benefits, and potential risks of the study, in conformity with the Code of Ethics of the World Medical Association (Declaration of Helsinki). The protocol and the methods applied in the study were approved by the Ethical Committee of the Faculty of Kinesiology, University of Split.

${ }^{1}$ BASKETBALL REFERENCE, http://www.basketball-reference.com/play-index/ plus/shot_finder.cgi 


\section{Protocol}

In the first session the participants performed a Yo-Yo Intermittent Recovery test level 1 (Yo-Yo IR1; Castagna et al., 2008b) to assess maximal heart rate (HRMAX). One week later the participants performed three randomized shooting testing sessions, with a 1-h rest plus warm-up between one session and the next one. All sessions consisted of 10 consecutive 3Ss (ball-Molten gf7, 600 gr.) from five different significant field spots just beyond the FIBA three-point line, i.e., about $7 \mathrm{~m}$ from the basket (similar to the NBA All-Star Weekend ThreePoint Contest shots ${ }^{2}$; Figure 1, two counter clockwise "laps") at three different heart rates (HR): rest $(0 \mathrm{HR})$, after warm-up with $\mathrm{HR}$ at $50 \% \mathrm{HRMAX}(50 \mathrm{HR}$; i.e., a reasonable post-warmup HR value; Garrett and Kirkendall, 2000), and 80\%HRMAX (80HR; i.e., a reasonable actual play HR value; McInnes et al., 1995), repeating the approach used by Padulo et al. (2015a). The same procedure was repeated 1 week later to evaluate the measures' reliability. More specifically, after a 15' standard warmup run, each participant threw 10 consecutive 3 Ss at different HRs (0HR-50HR-80HR). Each HR (continuously monitored with Cardio-Suunto ${ }^{\mathrm{TM}}$ ) was achieved by increasing the intensity of the shuttle running $(15+15 \mathrm{~m})$. Namely, the participants needed to run an average of 560 and $1600 \mathrm{~m}$ to achieve 50\%HRMAX and $80 \%$ HRMAX, respectively (i.e., to elicit a post-warm-up- and actual play-like fatigued state).

\section{Statistical Analysis}

Measures of central tendency and dispersion (mean $\pm S D$ and SE) were computed from the Yo-Yo IR1 and $0 \mathrm{HR}$, $50 \mathrm{HR}$, and $80 \mathrm{HR}$ (effective \%HRMAX and measured 3S\%) test results to summarize the data. Statistical analyses were performed with SPSS 17.0 (SPSS Inc., Chicago, IL). Distribution normality of the population was tested with the ShapiroWilk test, and homogeneity of variances was verified with Bartlett's test. The reliability of the $0 \mathrm{HR}, 50 \mathrm{HR}$, and $80 \mathrm{HR}$ tests was assessed by calculating the Intra-class Correlations Coefficient (ICC), according to the literature (Weir, 2005). Furthermore, a one-way within-participant's repeated-measures analysis of variance (ANOVA) was conducted to check for differences between the three test levels (0HR, 50HR, and $80 \mathrm{HR}$ ) with a post-hoc Bonferroni test. Effect sizes are presented as partial eta-squared $\left(\eta_{\mathrm{p}}^{2}\right)$ to determine the meaningfulness of the results. Level of statistical significance was set at a $P \leq 0.05$.

\section{RESULTS}

ICC showed a good reliability at 0HR (0.88), 50HR (0.91), and 80HR (0.94). Yo-Yo IR1-derived HRMAX was $195.6 \pm 6.1 \mathrm{bpm}$, obtained with a covered distance of $1,878 \pm 568 \mathrm{~m}$ and a final speed of $18.4 \pm 1.8 \mathrm{~km} / \mathrm{h}$. ANOVA confirmed differences of HR over the three administrated exercise conditions [Figure 2, top; $F_{(1.22)}=3405.722, P<0.0001, \eta^{2} p=0.990$ at $0 \mathrm{HR}$ $54.4 \pm 3.1 \mathrm{bpm}$, at 50HR $99.2 \pm 4.6 \mathrm{bpm}$, and at $80 \mathrm{HR}$ $155 \pm 6.5 \mathrm{bpm}]$. Similarly, ANOVA showed differences of

${ }^{2}$ NBA ALL STAR, http://www.nba-allstar.com/contests/3point/

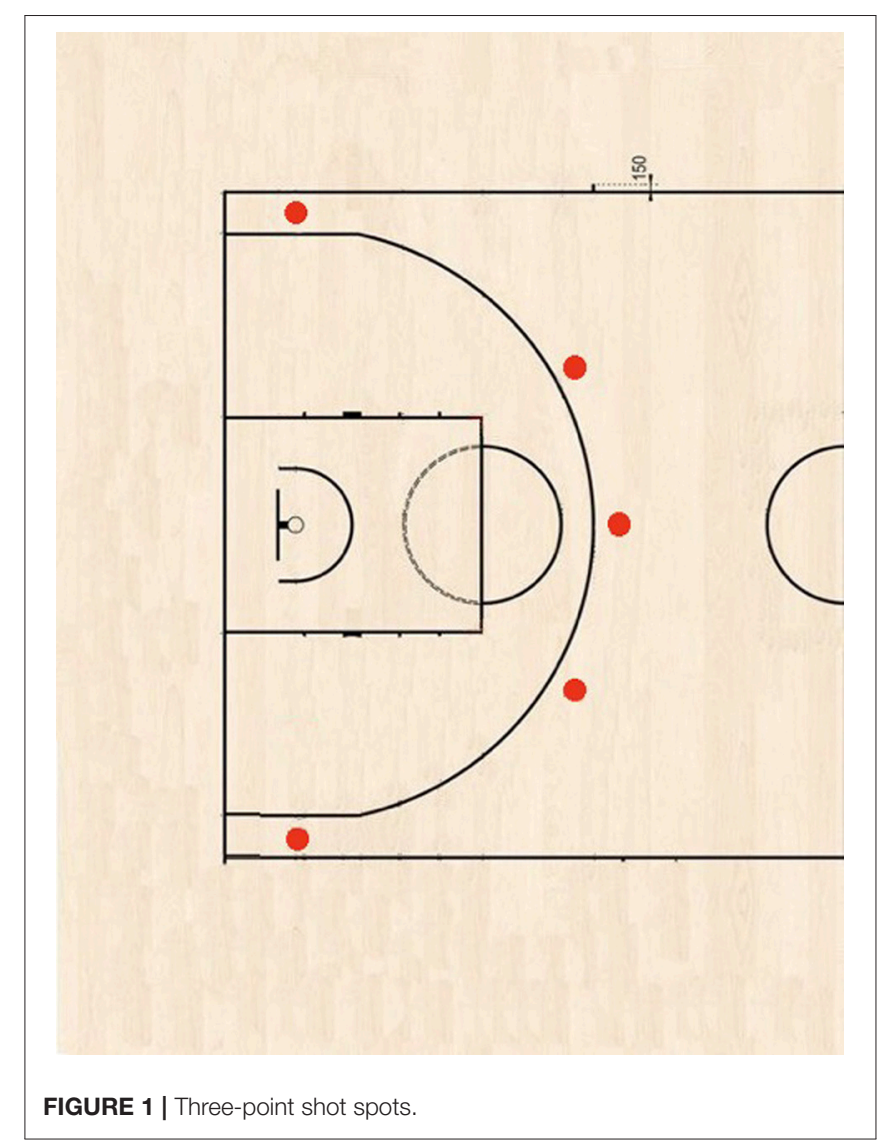

3S\% over the three exercise conditions [Figure 2, bottom; $\left.F_{(1.22)}=5.068, P=0.009, \eta^{2} p=0.131\right] .3 S \%$ in the three exercise conditions was $46.8 \pm 12.3 \%$ at $0 \mathrm{HR}, 41.3 \pm 10.7 \%$ at $50 \mathrm{HR}$, and $36.8 \pm 9.5 \%$ at $80 \mathrm{HR}$. The Bonferroni test did not show any significant $0 \mathrm{HR}-50 \mathrm{HR} 3 \mathrm{~S} \%(-15 \%, P=0.255)$ or $50 \mathrm{HR}-80 \mathrm{HR}(-12 \%, P=0.255)$ differences, whereas $80 \mathrm{HR}$ elicited significantly lower values of $3 \mathrm{~S} \%$ compared to $0 \mathrm{HR}$ $(-28 \%, P=0.007)$.

\section{DISCUSSION}

The major question of this study was whether there is any effect of different heart rates on successful three-point shot (3S) percentage $(3 S \%)$. Therefore, three different heart rates were elicited to investigate $3 \mathrm{~S} \%$ in young male basketball players: rest $(0 \mathrm{HR})$, after warm-up with heart rate (HR) at 50\%HRMAX (50HR), and 80\%HRMAX (80HR). According to the results, the main findings of the present study can be summarized as follows: (i) $50 \mathrm{HR}$ does not significantly decrease $3 \mathrm{~S} \%$, while on the other hand (ii) $80 \mathrm{HR}$ (i.e., intensity similar to that in real game-play; Ben Abdelkrim et al., 2010) significantly decreases $3 \mathrm{~S} \%$. The difference in $3 \mathrm{~S} \%$ between $0 \mathrm{HR}$ and $50 \mathrm{HR}$ was about $-15 \%$, whereas the difference between $0 \mathrm{HR}$ and $80 \mathrm{HR}$ was (significantly) $-28 \%$. The adverse effects of fatigue on performance and skills in basketball (Lyons et al., 2006; Ahmed, 2013; Padulo et al., 2015a) have been reported in studies on 


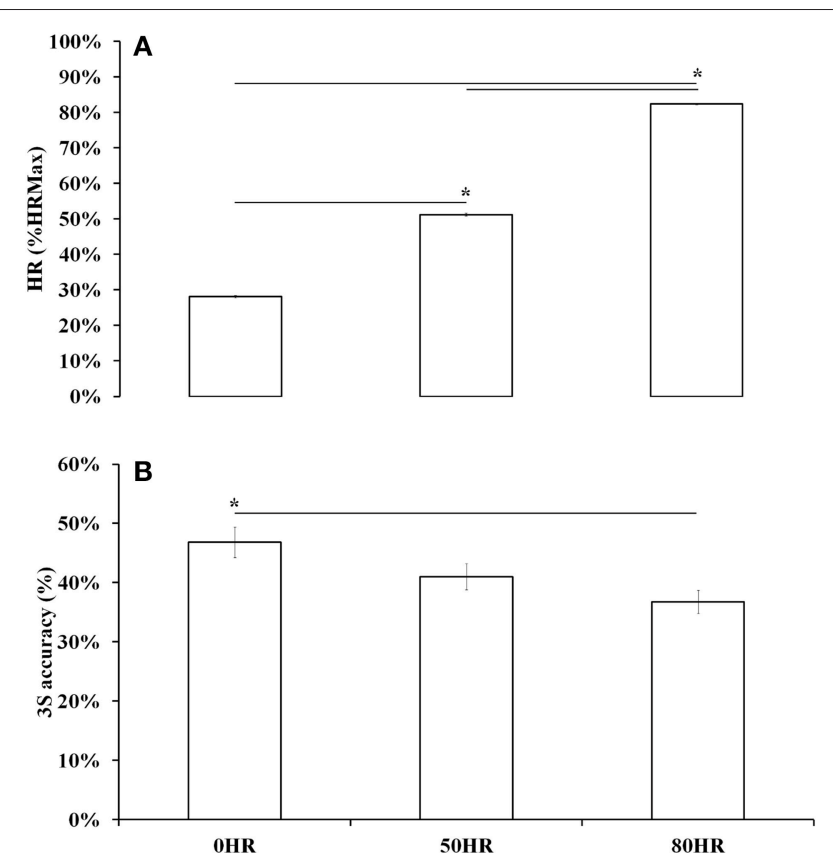

FIGURE 2 | Heart rate [HR (\%HRMax), A] and three-point shot percent accuracy [3S accuracy (\%), B], as mean $\pm S D$, over the three different testing conditions. ${ }^{*} P<0.05$

other sports, such as tennis (Rota et al., 2014), water polo (Royal et al., 2006), and soccer (Russell et al., 2011). The results obtained by Padulo et al. (2015a) on the effect of fatigue on successful basketball free-throw (FT) percentage (FT\%) are similar to the results of this study. Padulo et al. (2015a) reported that fatigue caused a decrease in FT\% from $-22 \%$ (between $80 \mathrm{HR}$ and $50 \mathrm{HR}$ ) to $-23 \%$ (between $80 \mathrm{HR}$ and $0 \mathrm{HR}$ ). Both FT and $3 \mathrm{~S}$ are complex basketball fundamentals, but are ecologically performed under very different game-play conditions. The FT is characterized by the fact that it is uncontested, which means that the shooter can be disturbed in a limited manner only by the opposing fans' movements and shouts (Padulo et al., 2015a). FT, which is a penalty for a player or team foul, contributes to from 15 to $21 \%$ of the total points scored during the game $\mathrm{e}^{3}$ As a predominantly anaerobic sport, most of the energy demand for basketball's highintensity activities, such as changes of direction, jumps, and shots, comes from the creatine phosphate system (CP; Metaxas et al., 2009). Yet a high fraction of maximum oxygen consumption is needed for recovery from such high-intensity activities (Tomlin and Wenger, 2001); it is therefore crucial for a player to recover in order to shoot successful FTs. A high level of aerobic fitness allows players to make a quick recovery after high-intensity activities, since muscle CP stores may be replenished within 30$40 \mathrm{~s}$ (Castagna et al., 2008a). It should be noted that in practice, a player is usually allowed about $30 \mathrm{~s}$ to shoot each FT (Padulo et al., 2015a).

${ }^{3}$ 2015-16 NBA season, TEAMRANKINGS, https://www.teamrankings.com/nba/ stat/percent- of-points-from-free-throws?date $=2016-06-20$.
In contrast, the $3 \mathrm{~S}$ shooter must complete a motor action that is far more complex in order to achieve his successful task, i.e., managing the related decision making under fatigue, in a very small time frame (often with the approach of the shot-clock end), and with the opposition of one or more defenders (Oudejans et al., 2005). In addition, potential off-court disturbance, such as visual distractions, can slightly change FT's kinematics but without decreasing its accuracy (Viggiano et al., 2014). Two- (2S) and three-point shots provide the most points contributions during a match, with total score percentages of $51-67 \%{ }^{4}$ and $16-35 \%^{5}$, respectively. $2 S$ and $3 S$ shots are the results of rapidly unfolding attack game plans, which very often include high-intensity movements. Given that the $3 \mathrm{~S}$ requires more coordination and strength, elite basketball players can incur potentially more negative effects due to fatigue than in sub-maximal performances requiring relatively less overall effort such as FT (Uygur et al., 2010). In basketball, shot performance requires a highly coordinated entire body, from feet to hands, and this is especially true for long-range shots and $3 \mathrm{Ss}^{6}$ (Okazaki and Rodacki, 2012). Explosive movements, featuring fundamentals such as the jump shot and 3S, make fatigue effects on shooting accuracy even more relevant. Threepoint shot accuracy decreases likely due to different reasons. We investigated its change (i.e., decrease) over changing metabolic demand (i.e., heart rate increase). What we found is at least a statistically significant link between the two signals. Like Padulo et al. (2015a) and Erculj and Supej (2009), we conclude that the training of shooting-be it FT or 3S-should be performed in conditions of from moderate-to-high fatigued state as well, so that an appropriate shooting technique can be preserved and will result in higher shooting accuracy during game-play.

\section{CONCLUSIONS}

As a practical implication for coaches and players, given that $50 \mathrm{HR}$ does not significantly decrease $3 \mathrm{~S} \%$ with respect to $0 \mathrm{HR}$, no preliminary warm-up is needed by the players before entering the game. In addition, since $80 \mathrm{HR}$ significantly decreases $3 \mathrm{~S} \%$ with respect to $50 \mathrm{HR}$, it has come to light that $3 \mathrm{~S}$ training in conditions of from moderate-to-high fatigued state is necessary to maintain high 3S\% during game-play. The results of this study should prompt future studies on the effects of fatigued state on shooting accuracy-not limited to $3 \mathrm{~S}$ but also on further (coordinationdriven!) fundamentals such as defense ones-that administers fatiguing protocols more ecological than basic shuttle running, e.g., real attack game plans. It would be interesting to use alternative metabolic intensity proxies, such as Karvonen's HR reserve (Karvonen et al., 1957) and/or rate of perceived exertion (Borg, 1982), as well. In terms of study limitations, it should be considered with caution that results were obtained under

\footnotetext{
${ }^{4}$ 2015-16 NBA season, TEAMRANKINGS, https://www.teamrankings.com/nba/ stat/percent- of- points-from-2-pointers?date $=2016-06-20$

${ }^{5}$ 2015-16 NBA season, TEAMRANKINGS, https://www.teamrankings.com/nba/ stat/percent- of-points-from-3-pointers?date $=2016-06-20$

${ }^{6}$ BASKETBALL REFERENCE, http://www.basketball-reference.com/play-index/ plus/shot_finder.cgi
} 
very controlled conditions, while real game-play takes place in a sometimes chaotic setting characterized by opponents of varied levels, numerous score differences, opposing fans' behavior, etc.). Basketball statistics have provided indications about other confounding factors as well, such as shooters shooting better when defended and shooters scoring several points over a stretch of time without any special reason" (i.e., the "hothand phenomenon or fallacy", "streaky" shooters, and/or the "Matthew effect"; Gilovich et al., 1985; Wardrop, 1995; Koehler and Conley, 2003; Arkes, 2010; Csapo and Raab, 2014; Csapo et al., 2015). Another study limitation, that could prompt further studies, is that we did not considered heart rate effect on $3 S \%$ in different playing positions and/nor elite adult players (we chose to investigate a typical whole youth team). In

${ }^{7} \mathrm{NBA}$, http://stats.nba.com

\section{REFERENCES}

Abd-Elfattah, H. M., Abdelazeim, F. H., and Elshennawy, S. (2015). Physical and cognitive consequences of fatigue: a review. J. Adv. Res. 6, 351-358. doi: 10.1016/j.jare.2015.01.011

Ahmed, T. (2013). The effect of upper extremity fatigue on grip strength and passing accuracy in junior basketball players. J. Hum. Kinet. 37, 71-79. doi: 10.2478/hukin-2013-0027

Arkes, J. (2010). Revisiting the hot hand theory with free throw data in a multivariate framework. J. Quant. Anal. Sports 6, 2. doi: 10.2202/1559-0410.1198

Barbieri, F. A., Rodrigues, S. T., Polastri, P. F., Barbieri, R. A., de Paula, P. H. A., Milioni, F., et al. (2017). High intensity repeated sprints impair postural control, but with no effects on free throwing accuracy, in under-19 basketball players. Hum. Mov. Sci. 54, 191-196. doi: 10.1016/j.humov.2017.04.010

Ben Abdelkrim, N., Castagna, C., Jabri, I., Battikh, T., El Fazaa, S., and El Ati, J. (2010). Activity profile and physiological requirements of junior elite basketball players in relation to aerobic-anaerobic fitness. J. Strength Cond. Res. 24, 2330-2342. doi: 10.1519/JSC.0b013e3181e381c1

Borg, G. A. (1982). Psychophysical bases of perceived exertion. Med. Sci. Sports Exerc. 14, 377-381. doi: 10.1249/00005768-198205000-00012

Borotikar, B. S., Newcomer, R., Koppes, R., and McLean, S. G. (2008). Combined effects of fatigue and decision making on female lower limb landing postures: central and peripheral contributions to ACL injury risk. Clin. Biomech. 23, 81-92. doi: 10.1016/j.clinbiomech.2007.08.008

Castagna, C., Abt, G., Manzi, V., Annino, G., Padua, E., and D’Ottavio, S. (2008a). Effect of recovery mode on repeated sprint ability in young basketball players. J. Strength Cond. Res. 22, 923-929. doi: 10.1519/JSC.0b013e31816a4281

Castagna, C., Impellizzeri, F. M., Rampinini, E., D’Ottavio, S., and Manzi, V. (2008b). The Yo-Yo intermittent recovery test in basketball players. J. Sci. Med. Sport 11, 202-208. doi: 10.1016/j.jsams.2007.02.013

Cortis, C., Tessitore, A., Lupo, C., Pesce, C., Fossile, E., Figura, F., et al. (2011). Inter-limb coordination, strength, jump, and sprint performances following a youth men's basketball game. J. Strength Cond. Res. 25, 135-142. doi: 10.1519/JSC.0b013e3181bde2ec

Csapo, P., Avugos, S., Raab, M., and Bar-Eli, M. (2015). How should "hot" players in basketball be defended? The use of fast-and-frugal heuristics by basketball coaches and players in response to streakiness. J. Sports Sci. 33, 1580-1588. doi: 10.1080/02640414.2014.999251

Csapo, P., and Raab, M. (2014). "Hand down, Man down." analysis of defensive adjustments in response to the hot hand in basketball using novel defense metrics. PLoS ONE 9:e114184. doi: 10.1371/journal.pone.0114184

Enoka, R. M., and Stuart, D. G. (1992). Neurobiology of muscle fatigue. J. Appl. Physiol. 72, 1631-1648.

Erculj, F., and Supej, M. (2009). Impact of fatigue on the position of the release arm and shoulder girdle over a longer shooting distance conclusion, different metabolic conditions also affect a relevant basketball fundamental such as $3 \mathrm{~S}$, and coaches and trainers should consider this when designing effective specific training regimes.

\section{AUTHOR CONTRIBUTIONS}

All authors listed have made a substantial, direct, and intellectual contribution to the work, and approved it for publication.

\section{ACKNOWLEDGMENTS}

The authors wish to thank all the players who volunteered in this study, and Ms. Dinah Olswang for English editing. No external financial support has been received.

for an elite basketball player. J. Strength Cond. Res. 23, 1029-1036. doi: 10.1519/JSC.0b013e3181a07a27

Faria, E. W., Parker, D. L., and Faria, I. E. (2005). The science of cycling: physiology and training - Part 1. Sports Med. 35, 285-312.

Garrett, W. E., and Kirkendall, D. T. (2000). Exercise and Sport Science. Philadelphia, PA: Lippincott Williams \& Wilkins.

Gilovich, T., Vallone, R., and Tversky, A. (1985). The hot hand in basketball: on the misperception of random sequences. Cogn. Psychol. 17, 295-314. doi: 10.1016/0010-0285(85)90010-6

Ibáñez, S. J., Feu, S., Cañadas, M., Parejo, I., and Cañadas, M. (2009). Shot differences between professional (ACB) and amateur (EBA) basketball teams. Multifactorial study. Rev. Psicol. Deport. 18, 313-317.

Kamandulis, S., Venckunas, T., Masiulis, N., Matulaitis, K., Balciūnas, M., Peters, D., et al. (2013). Relationship between general and specific coordination in 8- to 17-year-old male basketball players. Percept. Mot. Skills 117, 821-836. doi: 10.2466/25.30.PMS.117x28z7

Karvonen, M. J., Kentala, E., and Mustala, O. (1957). The effects of training on heart rate; a longitudinal study. Ann. Med. Exp. Biol. Fenn. 35, 307-315.

Knicker, A. J., Renshaw, I., Oldham, A. R., and Cairns, S. P. (2011). Interactive processes link the multiple symptoms of fatigue in sport competition. Sports Med. 41, 307-328. doi: 10.2165/11586070-000000000-00000

Koehler, J. J., and Conley, C. A. (2003). The 'Hot Hand' myth in professional basketball. J. Sport Exerc. Psychol. 25, 253. doi: 10.1123/jsep.25.2.253

Lyons, M., Al-Nakeeb, Y., and Nevill, A. (2006). The impact of moderate and high intensity total body fatigue on passing accuracy in expert and novice basketball players. J. Sports Sci. Med. 5, 215-227.

McInnes, S. E., Carlson, J. S., Jones, C. J., and McKenna, M. J. (1995). The physiological load imposed on basketball players during competition. J. Sports Sci. 13, 387-397. doi: 10.1080/02640419508732254

Metaxas, T. I., Koutlianos, N., Sendelides, T., and Mandroukas, A. (2009). Preseason physiological profile of soccer and basketball players in different divisions. J. Strength Cond. Res. 23, 1704-1713. doi: 10.1519/JSC.0b013e3181b3e0c5

Nikolaidis, P., Calleja-González, J., and Padulo, J. (2014). The effect of age on positional differences in anthropometry, body composition, physique and anaerobic power of elite basketball players. Sport Sci. Health 10, 225-233. doi: 10.1007/s11332-014-0198-5

Nikolaidis, P. T., Asadi, A., Santos, E. J., Calleja-González, J., Padulo, J., Chtourou, H., et al. (2015). Relationship of body mass status with running and jumping performances in young basketball players. Muscles Ligaments Tendons J. 5, 187-194. doi: 10.11138/mltj/2015.5.3.187

Noakes, T. D. (2012). Fatigue is a brain-derived emotion that regulates the exercise behavior to ensure the protection of whole body homeostasis. Front. Physiol. 3:82. doi: $10.3389 /$ fphys.2012.00082

Okazaki, V. H., and Rodacki, A. L. (2012). Increased distance of shooting on basketball jump shot. J. Sci. Med. Sport 11, 231-237. 
Okazaki, V. H., Rodacki, A. L., and Satern, M. N. (2015). A review on the basketball jump shot. Sports Biomech. 14, 190-205. doi: 10.1080/14763141.2015.10 52541

Ortega, E., Cárdenas, D., Sainz de Baranda, P., and Palao, J. M. (2006). Differences between winning and losing teams in youth basketball games (14-16 years old). Int. J. Appl. Sport Sci. 18, 1.

Oudejans, R. R., Koedijker, J. M., Bleijendaal, I., and Bakker, F. C. (2005). The education of attention in aiming at a far target: training visual control in basketball jump shooting. Int. J. Sport Exerc. Psychol. 3, 197-221. doi: 10.1080/1612197X.2005.9671767

Padulo, J., Attene, G., Migliaccio, G. M., Cuzzolin, F., Vando, S., and Ardigò, L. P. (2015a). Metabolic optimisation of the basketball free throw. J. Sports Sci. 33, 1454-1458. doi: 10.1080/02640414.2014. 990494

Padulo, J., Laffaye, G., Haddad, M., Chaouachi, A., Attene, G., Migliaccio, G. M., et al. (2015b). Repeated sprint ability in young basketball players: one vs. two changes of direction (Part 1). J. Sports Sci. 33, 1480-1492. doi: 10.1080/02640414.2014.992936

Rota, S., Morel, B., Saboul, D., Rogowski, I., and Hautier, C. (2014). Influence of fatigue on upper limb muscle activity and performance in tennis. J. Electromyogr. Kinesiol. 24, 90-97. doi: 10.1016/j.jelekin.2013. 10.007

Royal, K. A., Farrow, D., Mujika, I., Halson, S. L., Pyne, D., and Abernethy, B. (2006). The effects of fatigue on decision making and shooting skill performance in water polo players. J. Sports Sci. 24, 807-815. doi: 10.1080/02640410500188928

Russell, M., Benton, D., and Kingsley, M. (2011). The effects of fatigue on soccer skills performed during a soccer match simulation. Int. J. Sports Physiol. Perform. 6, 221-233. doi: 10.1123/ijspp.6.2.221
St Clair Gibson, A., Baden, D. A., Lambert, M. I., Lambert, E. V., Harley, Y. X., Hampson, D., et al. (2003). The conscious perception of the sensation of fatigue. Sports Med. 33, 167-176. doi: 10.2165/00007256-200333030-00001

Tomlin, D. L., and Wenger, H. A. (2001). The relationship between aerobic fitness and recovery from high intensity intermittent exercise. Sports Med. 31, 1-11. doi: 10.2165/00007256-200131010-00001

Uygur, M., Goktepe, A., Ak, E., Karabörk, H., and Korkusuz, F. (2010). The effect of fatigue on the kinematics of free throw shooting in basketball. J. Hum. Kinet. 24, 51-56. doi: 10.2478/v10078-010-0019-0

Viggiano, A., Chieffi, S., Tafuri, D., Messina, G., Monda, M., and De Luca, B. (2014). Laterality of a second player position affects lateral deviation of basketball shooting. J. Sports Sci. 32, 46-52. doi: 10.1080/02640414.2013.805236

Wardrop, R. L. (1995). Simpson's paradox and the hot hand in basketball. Am. Stat. 49, 24-28. doi: 10.1080/00031305.1995.10476107

Weir, J. P. (2005). Quantifying test-retest reliability using the intra-class correlation coefficient and the SEM. J. Strength Cond. Res. 19, 231-240. doi: $10.1519 / 15184.1$

Conflict of Interest Statement: The authors declare that the research was conducted in the absence of any commercial or financial relationships that could be construed as a potential conflict of interest.

Copyright (C) 2018 Ardigò, Kuvacic, Iacono, Dascanio and Padulo. This is an openaccess article distributed under the terms of the Creative Commons Attribution License (CC BY). The use, distribution or reproduction in other forums is permitted, provided the original author(s) and the copyright owner are credited and that the original publication in this journal is cited, in accordance with accepted academic practice. No use, distribution or reproduction is permitted which does not comply with these terms. 